\title{
Truth-Grounding and Transitivity
}

\author{
Tuomas E. Tahko \\ University of Helsinki
}

It is argued that if we take grounding to be univocal, then there is a serious tension between truthgrounding and one commonly assumed structural principle for grounding, namely transitivity. The primary claim of the article is that truth-grounding cannot be transitive. Accordingly, it is either the case that grounding is not transitive or that truth-grounding is not grounding, or both.

Keywords truthmaking; grounding; transitivity; relevance; discernment; dependence

DOI:10.1002/tht3.94

\section{Introduction}

Grounding is generally considered to be irreflexive, asymmetric, and transitive (Clark and Liggins 2012; Correia and Schnieder 2012). As an example of transitivity, we might say that the existence of a set is grounded in the existence of its members, and if its members include other sets, its existence is grounded in the existence of their members. Attempts to explicate the notion of ground in terms of entailment, supervenience, truthmaking, and different varieties of ontological dependence are familiar, but at least the first two fail to satisfy some of the structural principles of ground. Some varieties of ontological dependence may align with ground, but our primary interest is the use of grounding to explicate the notion of truthmaking. The idea of truth-grounding is familiar from recent literature on grounding and truthmaking: some entity $x$ grounds the truth of some proposition $p$ if $p$ is true in virtue of the existence of $x$ (Cameron forthcoming, p. 2). So, the entities which ground the truth of $p$ are $p$ 's truthmakers. ${ }^{1}$

Cameron (forthcoming, p. 3) goes on to specify that: 'The in virtue of relation is irreflexive, asymmetric and transitive. The converse of this relation is grounding: $<\mathrm{q}>$ grounds $<\mathrm{p}>$ just in case $<\mathrm{p}>$ is true in virtue of $<\mathrm{q}>$ '. Note that some proponents of grounding take it to be a relation, whereas some prefer an operational approach. ${ }^{2}$ The second, preferred for instance by Correia (2012) and Fine (2012a, 2012b), is neutral about the existence of grounds (sentences, facts, states of affairs ... ), whereas the first requires an account of the relata, the grounded and the grounding entities (cf. Rodriguez-Pereyra 2005). The relational approach seems, at least initially, more natural when it comes to truth-grounding. For instance, on an understanding of grounding as a relation between facts, we may define truth-grounding as follows: 
(TG) If the fact that $q$ exists helps ground the fact that $p$ is true, then $q$ helps ground the truth of $p$, i.e., $q$ is a (partial) truth-ground for $p$.

However, TG violates an important feature of traditional truthmaking theory, namely, truthmaking is generally understood to be a cross-categorical relation between an entity or entities and a truth, not merely a relation between facts (Armstrong 2004, p. 5). One might suggest that this is a mistake: if the truth-grounding account of truthmaking is correct, then truthmaking should not be considered cross-categorical at all. We will not attempt to settle this controversy here. Rather, for the sake of argument we will assume that TG captures the basic idea of truthmaking and examine whether it is compatible with transitivity. We suspect that it is not, which leads us to think that one of the three following claims must hold:

(1) Grounding is nontransitive.

(2) Truth-grounding is not grounding.

(3) 'Grounding' is not univocal: there are several varieties of grounding.

It is possible for (1) and (2) to hold at the same time, and also for (2) and (3). It is also possible to read (1) and (3) in such a way that they are compatible, namely, (1) could refer to some types of grounding. But our reading of (1) is absolute: it states that transitivity is not a structural principle of ground. We argue that the choice should be made between (1), (2), and (1) and (2). Point (3) is undesirable and would seem to corroborate recent claims about the multiplicity or even incoherence of 'grounding', making it an ambiguous expression (e.g. Daly 2012; Wilson 2013). If grounding comes in a number of different varieties, then what is common to these varieties? One might suggest that what is common is that they are all noncausal, but that would be a weak common denominator. So, the worry is that 'grounding' would then refer to a number of different types of dependence and (noncausal) explanation that do not even share a core set of structural principles, such as transitivity. Accordingly, we will rule out (3), and the same reasons lead us to rule out (2) and (3) and the combination of all three claims. ${ }^{3}$

This leaves us with three live options out of the seven possible combinations:

\begin{tabular}{lc}
\hline Options & LIVE/DEAD \\
\hline$(1)$ & LIVE \\
$(2)$ & LIVE \\
$(3)$ & DEAD \\
$(1)$ and (2) & LIVE \\
$(1)$ and (3) & DEAD \\
$(2)$ and (3) & DEAD \\
$(1),(2)$, and (3) & DEAD \\
\hline
\end{tabular}

Since we take 'grounding' to be univocal, i.e., there is only one variety of grounding, and read (1) in the absolute sense, just one successful example of grounding that is not transitive would affirm (1) — transitivity is either a universal structural principle for 
grounding or it never is. Schaffer (2012) has provided three such examples, but they have not been met very favourably — Litland (2013) and Raven (2013) address all of them in order to maintain the transitivity of grounding, successfully in our opinion. However, as Rosen (2010, p. 116) notes, the grounding relation 'is not obviously transitive', even though most authors, Rosen included, assume transitivity.

In the following section a brief survey of truth-grounding will be provided, after which it will be argued that truth-grounding cannot be transitive, whether or not it is considered to be a variety of grounding. We will not attempt to decide between the three live options here. The purpose of this article is to show that there is a tension between a commonly assumed structural principle for grounding — transitivity — and the view that truth-grounding is a species of grounding.

\section{Truth-grounding and grounding}

In this section, we attempt to demonstrate two things. First, truth-grounding is often considered to be subsumed under 'grounding' despite the problems pointed out above. Second, truth-grounding understood as a variety of grounding is often considered to be transitive.

Typical formulations of truth-grounding would seem to corroborate both of these claims. Cameron defines truthmaking in terms of a transitive in virtue of relation: 'Since $<\mathrm{r}>$ explains why $<\mathrm{q}>$ is true and $<\mathrm{q}>$ explains why $<\mathrm{p}>$ is true, there is a perfectly good sense in which $<\mathrm{r}>$ explains why $<\mathrm{p}>$ is true- so we say that $<\mathrm{p}>$ is true in virtue of $<r>$ ' (Cameron forthcoming, p. 3). Similarly, Correia (2011, pp. 3-5) takes transitivity to be one of the basic structural principles of grounding, which align nicely with what he takes to be the structural principles of truthmaking. ${ }^{4}$

It may seem that truth-grounding can be chained in the manner required by transitivity. Take the following example, adapted from Schnieder (2006, p. 33):

(i) Xanthippe became a widow.

The truth of (i) is grounded in the truth of a transitive chain of explanations:

(ii) Xanthippe became a widow, because Socrates died.

(iii) Socrates died, because he drank a cup of hemlock.

(iv) Socrates drank a cup of hemlock, because he was a man of principle.

Claim (ii) appears to be a straight-forward, legitimate grounding claim; the truth of the fact that Xanthippe became a widow is (fully) grounded in the fact that Socrates died. Claim (iii) involves a causal explanation rather than a truth-grounding claim, but it could be omitted from the chain of explanation, as it is (iv) that does most work in grounding the truth of (i): the fact that Socrates drank a cup of hemlock (which caused his death), is (at least partially) grounded in the fact that he was a man of principle and decided to sacrifice himself. Indeed, we might say that the fact that Socrates was sentenced to death in the first place is (partially) grounded in the fact that he was a man of principle. Accordingly, truth-grounding is not obviously incompatible with transitivity. 


\section{Truth-grounding and transitivity}

Truth-grounding is, by many, considered to be a form of grounding. It is also, at least by some, considered to be transitive. In this section we will argue that truth-grounding cannot be transitive.

The argument that follows relies on a qualified version of a principle that Raven (2013) calls relevance. Raven discusses - and dismisses - a generic version of this principle as something that might be behind Schaffer's (2012) counterexamples to the transitivity of grounding, but our version is restricted to truth-grounding, call it truth-grounding relevance:

(TGR) If the fact that $q$ is not relevant to the truth of proposition $p$, then it is not the case that $q$ helps ground the truth of $p$.

The sense of relevance captured by TGR can be distinguished from a sense of relevance which seems central to all grounding claims. With regard to grounding in general, it is plausible that the converse of TGR holds: if the fact that $q$ does not help ground the truth of $p$, then $q$ is not relevant to the truth of $p$. In many cases, both TGR and its converse would seem to hold. For instance, Rodriguez-Pereyra (2006, p. 972) suggests that the fact that Saturn is a planet does not contribute to the truth, i.e., does not help ground the truth, of the proposition 'Peter is a man' - the fact in question is irrelevant to the truth of the proposition. In this case, it is perfectly clear that the fact about Saturn is irrelevant to the truth of 'Peter is a man' and that both TGR and its converse will apply, but the notion of relevance applicable to truth-grounding can be specified further.

Consider the redness of a particular rose-an example that is becoming something of a classic in the literature. The following example is closest to Trogdon's (2013a) version, which is adapted from Schaffer. Let us assume for the purposes of this argument that there is a redness mode/trope, dependent on a particular substance, the rose. We might say that the fact that the rose is red is partially grounded in the fact that the rose reflects light with a particular wavelength. But given the substance/mode ontology, we might also say that the fact that the rose is red is partially grounded in the fact that the rose exists. Certainly, there is some sense of relevance in which the rose's existence is relevant to the truth of the rose having a particular redness, for the particular redness mode is dependent on the rose's existence. Yet, Trogdon, expressing agreement with Schnieder (2006), proposes that the rose's existence adds nothing of relevance to the explanans, specifying that 'the existence of the particular redness is fully grounded in the fact that the rose is a particular shade of red' (Trogdon 2013a, p. 479). ${ }^{5}$ Whether or not Trogdon is right about this example, here we have a case where two senses of relevance can be distinguished, one of which is clearly stricter. We wish to remain neutral about which sense of relevance is associated with grounding in general. ${ }^{6}$ In any case, it seems clear that there are at least two different types of intuitive reaction that one might have in scenarios such as the one that Trogdon puts forward - these reactions are based precisely on the interpretation of the relevance-link required for a valid grounding claim. 
What we insist is that the sense of relevance associated with TGR must be of the stricter type. Note that if there were two different senses of relevance at play, then it appears that truth-grounding relevance would not be exhausted by fact $q$ fully or partially grounding fact $p$. Hence, assuming univocalism regarding 'relevance' associated with grounding in general, truth-grounding would not be a variety of grounding. Be that as it may, the sense of relevance associated with TGR is not compatible with transitivity, or so we will attempt to demonstrate with the following example.

Consider the proposition 'There exists a particular bottle of beer, $b$.' What is relevant to the truth of this proposition? We might state the following grounding claims:

(v) The fact that a particular bottle of beer, $b$, exists is partially grounded in the fact that $b$ has a stable macrophysical structure.

(vi) The fact that $b$ has a stable macrophysical structure is partially grounded in the fact that the Pauli Exclusion Principle holds.

Both of these grounding claims may be considered plausible. Regarding (v), we wouldn't think that the bottle of beer exists if it didn't have roughly the sort of properties - all of them macrophysical - that we expect from a bottle of beer, such as being rigid (assuming that it is a glass bottle), and containing a tasty, usually alcoholic and thirst quenching beverage. All of the macrophysical properties of $b$ are grounded in certain microphysical properties, the causal features of the constituent microphysical particles in particular, which together produce a stable macrophysical structure. This is not to say that all necessary conditions are grounds, but (v) seems as plausible a candidate as any. As to (vi), the Pauli Exclusion Principle-sometimes said to be responsible for the space-occupying behaviour of all matter-is one of the most important physical principles regarding the stability of matter (on this, see Tahko 2012). ${ }^{7}$ So, there are good reasons to think that the Pauli Exclusion Principle is at least a partial ground for many claims concerning macrophysical stability.

Now, by transitivity, we get:

(vii) The fact that $b$ exists is partially grounded in the fact that the Pauli Exclusion Principle holds.

This grounding claim may not seem problematic at first glance. It seems perfectly plausibly that the existence of $b$ is partially grounded in the physical principles that govern microphysical facts. This line of thought follows Cameron (forthcoming, p. 3), according to whom the 'ultimate ground' is only reached with the absolutely fundamental truths; in this case general principles like the Pauli Exclusion Principle. ${ }^{8}$ In this sense, it seems that the Pauli Exclusion Principle is relevant to $b$ 's existence, since $b$ 's existence is indeed dependent on it. Given the conditional nature of the current project, we can leave it open whether (vii) is a valid grounding claim - it is not our aim here to establish that (vii) cannot be a valid grounding claim. What we do aim to establish is that the relevance-link in (vii) is not of the strict type, analogously to the case of the rose and its particular redness. Accordingly, (vii) does not satisfy the strict sense of TGR and hence it is not a valid truth-grounding claim. Whether or not (vii) and similar cases constitute 
valid grounding claims of some other type is left open, but there are reasons to think that they do not constitute valid truth-grounding claims.

Why does (vii) not constitute a valid truth-grounding claim? Because the chaining from (v) to (vii) does not satisfy the explanatory requirement of truthmaking. Describing the fundamental laws governing the microstructure of $b$ in no way contributes to answering the question: What makes it true that 'There exists a particular bottle of beer, $b$ '? A central task of truthmaking theory is to provide an explanation as to what makes one rather than another proposition true, call it the discernment task of truthmaking theory (Armstrong 2004, p. 18; Lowe 2009, p. 201). This task is not helped by listing fundamental laws that partially ground the existence of all macrophysical objects whatsoever. ${ }^{9}$ The point is that while listing such laws is relevant for highly general question such as 'How can any macrophysical objects exist at all?' they do not offer anything that would be relevant strictly to the case of (vii). We could compare the situation to the familiar case of 'the world' making true any proposition whatsoever (Schaffer 2010). While there is a sense in which this is true, there is also a sense in which it is clearly inadequate-because imprecise and nondiscerning - to answer 'the world', to any and all truth-grounding questions. There is a similar, even though slightly less radical lack of precision in (vii). Of course, one could reject the discernment task of truthmaking theory, perhaps following Schaffer. But abandoning such a central desideratum of truthmaking theory would take away much of what is supposed to be appealing about it in the first place. Our claim here should be understood as conditional: if one accepts the discernment task, then the grounding claim in (vii) loses much of its relevance and hence fails to satisfy TGR.

To be more precise about what the discernment task amounts to, we might say that specifying the immediate ground(s) of the truth of a proposition is the explanatory 'ultimate ground' and that is where truth-grounding terminates. ${ }^{10}$ Moreover, there appears to be a shift in the type of dependence associated with immediate and mediate grounds for the truth of a proposition. The grounding claim in (v) concerning the stability of the macrophysical structure of the bottle — that very bottle - has a high level of discernment. Due to being highly discerning, (v) respects the explanatory goals of truthmaking theory. But the grounding claim in (vii) appears to have lost the strict explanatory relevance-link, as it fails to discern between any macrophysical objects.

To elaborate (even at the risk of repetition): a particular bottle of beer, $b$, depends for its existence on certain microphysical facts. We might say that the dependence at hand concerns the part-whole relation between $b$ and its constituent molecules. However, the bottle does not depend for its existence on the very molecules which happen to constitute it, even though it depends for its existence on the existence of some molecules. This is all plausible, but it does not capture the sense of truth-grounding relevance that we have been dealing with. What makes true the proposition 'There exists a particular bottle of beer, $b$.' is that $b$ is able to fulfil the role that it has in that proposition. To fulfil this role, the bottle must exist, and its existence depends on there being a stable arrangement of some molecules, which in turn depends on the Pauli Exclusion Principle. But once it has been determined that the bottle exists, there is nothing more that is needed to establish 
the truth of the proposition 'There exists a particular bottle of beer, $b$ ' — the story about microphysics adds nothing of relevance in the sense of TGR. ${ }^{11}$

Accordingly, we suggest defining truth-grounding relevance in terms of the closest explanans - the immediate ground(s). We conclude that truth-grounding cannot be transitive. If this is right, then it is either the case that grounding is not transitive or that truth-grounding is not grounding, or both.

\section{Acknowledgements}

Thanks to Donnchadh O'Conaill, Benjamin Schnieder, Alexander Skiles, and Kelly Trogdon for helpful comments on earlier versions of this article. Thanks also to the audience at the 2nd NIP Early Career Conference in Aberdeen, especially Douglas Edwards. The research for this paper was made possible by a grant from the Academy of Finland.

\section{Notes}

1 We should distinguish between full and partial truth-grounds here, following Fine (2012a, p. 3 ), who suggests that $P$ and $Q$ together fully ground $P \& Q$, and $P$ and $Q$ are each a partial ground for $P \& Q$.

2 For a discussion of this distinction, see Trogdon (2013b).

3 Trogdon (2013b) also assumes univocalism, suggesting that it is a reasonable starting point. To be precise, univocalism suggests that 'grounding' should be considered in terms of a single notion of dependence (which may or may not be analysable in terms of other notions, depending on the type of univocalism). Trogdon also associates this type of univocalism with Rosen (2010).

4 More precisely, transitivity entails one of the structural principles of truthmaking, which Correia (2011, p. 4) calls the Grounding Thesis.

5 This example has been met with some scepticism by commentators. It is admittedly controversial, but this only serves to corroborate the claim being made, namely, that there seem to be (at least) two different types of relevance that may be associated with grounding claims.

6 If grounding in general requires the stricter type of relevance, then both TGR and its converse will hold for all grounding claims. Note also that, assuming univocalism, there should be only one sense of relevance associated with grounding in general.

7 More precisely, the Pauli Exclusion Principle states that two fermions in a closed system cannot be in the same quantum state at the same time.

8 The same line of thought is reflected by Fine (2012b, p. 44), although he recognizes the problem that transitivity raises for truthmaking. Note also that the case could certainly be made with other 'fundamental' laws of physics as well.

9 The Pauli Exclusion Principle is an excellent candidate for such a general law, but for the purposes of this paper it is not necessary to discuss the physics in any detail—let us simply assume that there are some general physical principles that are necessary for the forming of macrophysical objects.

10 Following Fine (2012b), we can distinguish between immediate and mediate ground, where an immediate ground is a direct ground and a mediate ground is an indirect ground that 
must be mediated through other grounding relationships — only the notion of mediate ground is transitive.

11 Commentators have pointed out that the case, if correct, might very well generalize to other types of grounding, beyond truth-grounding. In fact, the worry has been expressed that unless this is the case, then the point might simply amount to there being an epistemic sense to TGR, whereas the sense of relevance for grounding claims in general ought to be ontological. This would seem to imply that truth-grounding is not grounding, insofar as we wish to maintain that grounding is an ontological rather than an epistemological notion. This issue cannot be fully addressed here, but the notion of relevance and its apparent epistemic connotations clearly deserve further attention.

\section{References}

Armstrong, D. M. Truth and Truthmakers. Cambridge: Cambridge University Press, 2004.

Cameron, R. "Truthmakers," in The Oxford Handbook of Truth, edited by M. Glanzberg. Oxford: Oxford University Press, forthcoming. Retrieved from http://www.personal.leeds.ac.uk/ phlrpc/truthmakers\%20handbook\%20truth.pdf on March 1, 2013.

Clark, M. J. and D. Liggins. "Recent Work on Grounding.” Analysis 72.4 (2012): 812-23.

Correia, F. "From Grounding to Truth-Making: Some Thoughts," in Philosophical papers dedicated to Kevin Mulligan, edited by A. Reboul. Genève, 2011. Retrieved from http://www.philosophie.ch/kevin/festschrift/Correia-paper.pdf.

."Grounding and Truth-Functions." Logique \& Analyse 211 (2012): 251-79.

Correia, F. and B. Schnieder, (eds). Metaphysical Grounding: Understanding the Structure of Reality. Cambridge: Cambridge University Press, 2012.

Daly, C. "Scepticism About Grounding," in Metaphysical Grounding, edited by F. Correia and B. Schnieder. Cambridge: Cambridge University Press, 2012, 81-100.

Fine, K. “The Pure Logic of Ground.” The Review of Symbolic Logic 5.1 (2012a): 1-25. . "Guide to Ground," in Metaphysical Grounding, edited by F. Correia and B. Schnieder. Cambridge: Cambridge University Press, 2012b, 37-80.

Litland, J. "On Some Counterexamples to the Transitivity of Grounding." Essays in Philosophy 14.1 (2013, Article 3): 19-32.

Lowe, E. J. "An Essentialist Approach to Truth-making," in Truth and Truth-Making, edited by E. J. Lowe and A. Rami. Stocksfield: Acumen, 2009, 201-16.

Raven, M. “Is Ground a Strict Partial Order?” American Philosophical Quarterly 50.2 (2013): 191-9.

Rodriguez-Pereyra, G. "Why Truthmakers?," in Truthmakers: The Contemporary Debate, edited by H. Beebee and J. Dodd. Oxford: Oxford University Press, 2005, 17-31.

. "Truthmaking, Entailment, and the Conjunction Thesis." Mind 115.460 (2006): 957-82.

Rosen, G. "Metaphysical Dependence: Grounding and Reduction," in Modality: Metaphysics, Logic, and Epistemology, edited by B. Hale and A. Hoffman. Oxford: Oxford University Press, 2010, 109-35.

Schaffer, J. "The Least Discerning and Most Promiscuous Truthmaker." Philosophical Quarterly 60.269 (2010): 307-24.

_. "Grounding, Transitivity, and Contrastivity," in Metaphysical Grounding, edited by F. Correia and B. Schnieder. Cambridge: Cambridge University Press, 2012, 122-38.

Schnieder, B. “Truth-Making Without Truth-Makers." Synthese 152.1 (2006): 21-46. 
Tahko, T. E. “Boundaries in Reality.” Ratio 25.4 (2012): 405-24.

Trogdon, K. “Grounding: Necessary or Contingent?” Pacific Philosophical Quarterly 94.4 (2013a): $465-85$.

. "An Introduction to Grounding," in Dependence: Basic Philosophical Concepts, edited by M. Hoeltje, B. Schnieder, and A. Steinberg. Munich: Philosophia Verlag, 2013b, 97-122.

Wilson, J. "No Work for a Theory of Grounding." Unpublished manuscript (2013). Retrieved from http://individual.utoronto.ca/jmwilson/NWfaTG.doc on May 1, 2013. 